\title{
Selective lithiation of 1-chloro-n-phenylsulfanylalkanes
}

\author{
Abdeslam Abou, Francisco Foubelo, and Miguel Yus* \\ Departamento de Química Orgánica and Instituto de Síntesis Orgánica (ISO), Facultad de \\ Ciencias, Universidad de Alicante, Apartado 99, E-03080 Alicante, Spain \\ E-mail:yus@ua.es
}

This paper is dedicated to Professor Lutz F. Tietze on occasion of his $65^{\text {th }}$ anniversary

\begin{abstract}
The reaction of different 1-chloro-n-phenylsulfanylalkanes 5 with 2 equivalents of lithium naphthalene at $-78{ }^{\circ} \mathrm{C}$ followed by addition of a carbonyl compound $\left[\mathrm{Bu}^{t} \mathrm{CHO}, \mathrm{Et}_{2} \mathrm{CO}\right.$, $\left(\mathrm{CH}_{2}\right)_{5} \mathrm{CO}$ ] leads, after hydrolysis with water, to the expected sulfanyl alcohols 8 through a selective lithiation of the carbon-chlorine bond. When an excess of lithium (1:4 molar ratio) is added to the reaction mixture before the hydrolysis and the system is allowed to reach $-50{ }^{\circ} \mathrm{C}$ during $1.5 \mathrm{~h}$, lithiation of the remaining carbon-sulfur bond takes place. The addition of a second carbonyl compound $\left(\mathrm{Bu}^{t} \mathrm{CHO}, \mathrm{PhCHO}\right)$, followed by hydrolysis, gives differently substituted diols 3 .
\end{abstract}

Keywords: Lithium naphthalene, chlorine-lithium exchange, sulfur-lithium exchange, electrophilic substitution, diols

\section{Introduction}

The reaction of dilithium compounds ${ }^{1}$ with electrophiles allows the preparation of difunctionalized molecules in a single synthetic operation. The stability of these intermediates, which exhibit fascinating structures, depends mainly on the relative position of the lithium atoms and also on the hybridization of the carbon atoms bonded to the metal. In general, they are accessible by applying the same methodologies as for single organolithium compounds, ${ }^{2}$ such as deprotonation reactions, halogen-lithium exchange, reductive cleavage of ethers and thioethers with lithium metal, and transmetallation process principally. The halogen-lithium exchange is probably the most commonly used method to generate organolithium intermediates and among thioethers, phenylthioethers undergo reductive cleavage lithiation under smooth reaction conditions by using lithium metal and either a stoichiometric ${ }^{3}$ or catalytic ${ }^{4}$ amount of an arene as electron carrier. Recently, we have reported the chemoselective monolithiation of different 1- 
bromo-n-chloroalkanes $\mathbf{1}(\mathrm{X}=\mathrm{Br})$ in the presence of a carbonyl compound to give functionalized alcohols 2. ${ }^{5}$ This process has to be performed at low temperature using a stoichiometric amount of the lithiating reagent and in the presence of the electrophile (Barbier-type reaction conditions $^{6}$ ) in order to avoid the decomposition of the highly reactive 1-chloro-n-lithioalkane intermediate initially formed. It was also possible to perform a sequential double lithiation reaction with electrophiles to prepare non-symmetrically and symmetrically substituted diols $\mathbf{3}$ and $4,^{7}$ respectively (Scheme 1). However, functionalized alcohols $\mathbf{2}$ and diols $\mathbf{3}$ were not accessible through this methodology for derivatives with $\mathrm{n}=0$ and 1 due to the previously known instability of the initially formed organolithium intermediate. In the context of our continuing interest in the dilithiation of different $1, n$ - difunctionalized compounds, we report here the selective mono- and dilithiation of a number of 1-chloro-n-phenylsulfanylalkanes ${ }^{8}$ and their use as dianionic synthetic equivalents in the reaction with different carbonyl compounds as electrophiles.

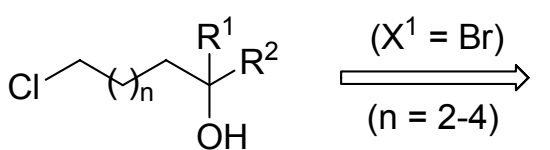

2

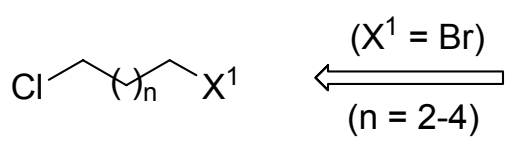

1<smiles>[R]C([R])(O)C[14CH]=[14CH]C[14C]([R])([R])O</smiles>

3

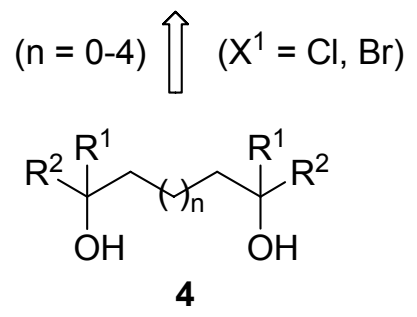

Scheme 1. Selective lithiation of 1,n-dihaloalkanes.

\section{Results and Discussion}

The reaction of 1-chloro-n-phenylsulfanylalkenes 5 (easily prepared from the corresponding 1bromo-n-chloroalkanes, $\mathbf{1}$, by reaction with sodium thiophenolate in methanol at room temperature) with two equivalents of lithium naphthalene in $\mathrm{THF}$ at $-78^{\circ} \mathrm{C}$ for $30 \mathrm{~min}$, followed by addition of a variety of carbonyl compounds $\left[\mathrm{R}^{1} \mathrm{R}^{2} \mathrm{CO}=\mathrm{Bu}^{t} \mathrm{CHO}, \mathrm{Et}_{2} \mathrm{CO},\left(\mathrm{CH}_{2}\right)_{5} \mathrm{CO}\right]$ as electrophiles, and final hydrolysis with water, gave the corresponding phenylsulfanylalcohols 8 (Scheme 2 and Table 1). 


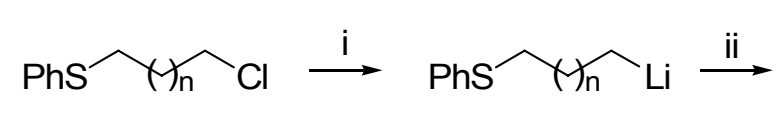

5

6

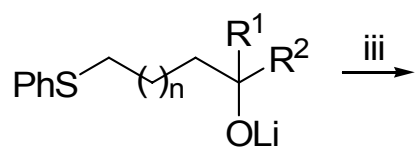

7<smiles>[R]C([R])(O)CC=CCSc1ccccc1</smiles>

8

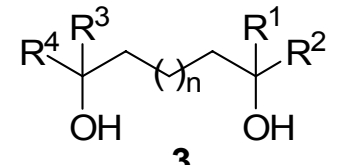

Scheme 2. Reagents and conditions: (i) $\mathrm{LiC}_{10} \mathrm{H}_{8}$, THF, $-78^{\circ} \mathrm{C}$; (ii) $\mathrm{R}^{1} \mathrm{R}^{2} \mathrm{CO},-78^{\circ} \mathrm{C}$; (iii) $\mathrm{H}_{2} \mathrm{O}$, -78 to $20{ }^{\circ} \mathrm{C}$; (iv) $\mathrm{Li}$, THF, -78 to $-50{ }^{\circ} \mathrm{C}$; (v) $\mathrm{R}^{3} \mathrm{R}^{4} \mathrm{CO},-50{ }^{\circ} \mathrm{C}$; (vi) -50 to $20^{\circ} \mathrm{C}$.

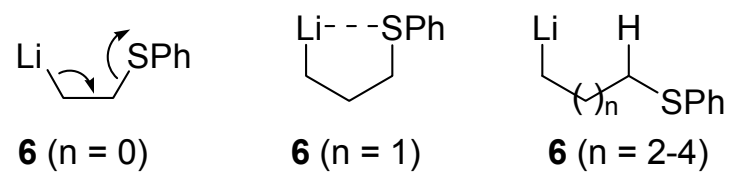

Chart 1. Organolithium intermediates 6.

Table 1. Selective monolithiation of 1-chloro-n-phenylsulfanylalkanes 5. Preparation of alcohols 8

\begin{tabular}{|c|c|c|c|c|c|}
\hline \multirow[b]{2}{*}{ Entry } & \multirow[b]{2}{*}{$\mathrm{n}$} & \multirow[b]{2}{*}{$\mathrm{R}^{1} \mathrm{R}^{2} \mathrm{CO}$} & \multicolumn{3}{|c|}{ Product $^{\mathrm{a}}$} \\
\hline & & & No. & Structure & Yield $(\%)^{b}$ \\
\hline 1 & 1 & $\mathrm{Bu}^{t} \mathrm{CHO}$ & $8 \mathbf{a}$ & & 81 \\
\hline 2 & 1 & $\left(\mathrm{CH}_{2}\right)_{5} \mathrm{CO}$ & $8 b$ & & 88 \\
\hline 3 & 2 & $\mathrm{Et}_{2} \mathrm{CO}$ & $8 c$ & & 36 \\
\hline 4 & 3 & $\mathrm{Et}_{2} \mathrm{CO}$ & $8 d$ & & 35 \\
\hline 5 & 4 & $\mathrm{Et}_{2} \mathrm{CO}$ & $8 e$ & & 44 \\
\hline
\end{tabular}

${ }^{\text {a }}$ All products 8 were $>95 \%$ pure (GLC and/or $300 \mathrm{MHz}{ }^{1} \mathrm{H}$ NMR) and were fully characterized by spectroscopic means (IR, ${ }^{1} \mathrm{H}$ and ${ }^{13} \mathrm{C} \mathrm{NMR}$, and LR and HR mass spectrometry). ${ }^{\mathrm{b}}$ Isolated yields of compounds $\mathbf{8}$ after column chromatography (silica gel, hexane/ethyl acetate). 
All attempts to perform the selective monolithiation of 1-chloro-2-phenylsulfanylethane $(\mathbf{5}, \mathrm{n}$ $=0)$ failed because of the instability of the resulting organolithium intermediate $6(n=0)$, which decomposes immediately through a $\beta$-elimination process to give ethylene (Chart 1$)$. The expected phenylsulfanylalcohols $8(n=0)$ were neither isolated nor detected by tandem MS/GC. Very good yields were obtained starting from 1-chloro-3-phenylsulfanylpropane $(\mathbf{5}, \mathrm{n}=1$, Table 1, entries 1 and 2). That indicates that intermediate 6 with $n=1$ (Chart 1) is quite stable, probably due to an intramolecular coordination of the metal with the sulfur atom ${ }^{9}$ which avoids the elimination process. Surprisingly, yields were considerably lower for the rest of 1-chloro-nphenylsulfanylalkenes 5 (Table 1, entries 3-5). An explanation for these results could be that intermediates 6 with $n=2-4$ (Chart 1) partially decompose by an intra- or intermolecular proton abstraction due to the acidity of the hydrogen atoms at the $\alpha$-position respect to the sulfur atom.

We also studied the introduction of two different electrophiles in a one-pot process taking advantage of the controlled monolithiation of the starting 1-chloro-n-phenylsulfanylalkenes 5 . Thus, alcoholate 7 , which was obtained by selective monolithiation of $5(n=1-4)$ followed by reaction with a first carbonyl compound as electrophile $\left\{\mathrm{R}^{1} \mathrm{R}^{2} \mathrm{CO}=\mathrm{Me}_{2} \mathrm{CO}, \mathrm{Et}_{2} \mathrm{CO}\right.$, $\left[\mathrm{Me}\left(\mathrm{CH}_{2}\right)_{4}\right]_{2} \mathrm{CO},\left[\mathrm{Me}\left(\mathrm{CH}_{2}\right)_{4}\right]_{2} \mathrm{CO},\left(\mathrm{CH}_{2}\right)_{5} \mathrm{CO},\left(\mathrm{CH}_{2}\right)_{5} \mathrm{CO},\left(\mathrm{CH}_{2}\right)_{7} \mathrm{CO},(-)$-menthone , was again lithiated at temperatures between -78 and $-50{ }^{\circ} \mathrm{C}$ by addition of an excess of lithium powder (1:4 molar ratio) to the reaction mixture to give the corresponding functionalized organolithium intermediate $^{10}$ 9. Subsequent addition of a second carbonyl compound $\left\{\mathrm{R}^{3} \mathrm{R}^{4} \mathrm{CO}=\mathrm{Bu}{ }^{t} \mathrm{CHO}\right.$, $\mathrm{PhCHO}$ ) at $-50{ }^{\circ} \mathrm{C}$, followed by hydrolysis with water at temperatures ranging between -50 and $20^{\circ} \mathrm{C}$ gave the corresponding diols 3 (Scheme 1 and Table 2).

The best yields of diols $\mathbf{3}$ were obtained starting from 1-chloro-3-phenylsulfanylpropane $\mathbf{5}$ (n $=1$, Table 2, entries 1-7). However, yields were considerably lower in the case of chlorothioethers 5 with $n=2-4$ (Table 2, entries 8-10) due to the instability of the corresponding organolithium intermediate $\mathbf{6}$ as commented above. The use of two prostereogenic carbonyl compounds such as (-)-menthone and pivalaldehyde afforded a 4:1 diastereomeric mixture of the expected diol $\mathbf{3 g}$ (Table 2, entry 7). ${ }^{11}$

In conclusion, we report here the selective monolithiation of 1-chloro-nphenylsulfanylalkanes 5 by using two equivalents of lithium naphthalene at $-78{ }^{\circ} \mathrm{C}$ as the lithiating reagent. More interesting is the sequential double lithiation followed by reaction with two different electrophiles, which allows the preparation of unsymmetrically substituted diols 3. We found especially interesting this methodology for the preparation of 1,5-diols which are not accessible through other methodologies, for instance, starting from dihalogenated compounds. 
Table 2. Sequential double lithiation of 1-chloro-n-phenylsulfanylalkanes 5. Preparation of diols 3

\begin{tabular}{|c|c|c|c|c|c|c|}
\hline \multirow[b]{2}{*}{ Entry } & \multirow[b]{2}{*}{$\mathrm{n}$} & \multicolumn{2}{|c|}{ Electrophiles } & \multicolumn{3}{|c|}{ Product $^{\mathrm{a}}$} \\
\hline & & $\mathrm{R}^{1} \mathrm{R}^{2} \mathrm{CO}$ & $\mathrm{R}^{3} \mathrm{R}^{4} \mathrm{CO}$ & No. & Structure & Yield $(\%)^{b}$ \\
\hline 1 & 1 & $\mathrm{Me}_{2} \mathrm{CO}$ & $\mathrm{Bu}^{t} \mathrm{CHO}$ & $\mathbf{3 a}$ & & 60 \\
\hline 2 & 1 & {$\left[\mathrm{Me}\left(\mathrm{CH}_{2}\right)_{4}\right]_{2} \mathrm{CO}$} & $\mathrm{Bu}^{t} \mathrm{CHO}$ & $\mathbf{3 b}$ & & 77 \\
\hline 3 & 1 & {$\left[\mathrm{Me}\left(\mathrm{CH}_{2}\right)_{4}\right]_{2} \mathrm{CO}$} & $\mathrm{PhCHO}$ & $3 \mathbf{c}$ & & 80 \\
\hline 4 & 1 & $\left(\mathrm{CH}_{2}\right)_{5} \mathrm{CO}$ & $\mathrm{Bu}^{t} \mathrm{CHO}$ & 3d & & 74 \\
\hline 5 & 1 & $\left(\mathrm{CH}_{2}\right)_{5} \mathrm{CO}$ & $\mathrm{PhCHO}$ & $3 \mathbf{e}$ & & 68 \\
\hline 6 & 1 & $\left(\mathrm{CH}_{2}\right)_{7} \mathrm{CO}$ & $\mathrm{PhCHO}$ & 3f & & 72 \\
\hline 7 & 1 & (-)-menthone & $\mathrm{Bu}^{t} \mathrm{CHO}$ & $3 g$ & & $41^{\mathrm{c}}$ \\
\hline 8 & 2 & $\mathrm{Et}_{2} \mathrm{CO}$ & PhCHO & $3 \mathbf{h}$ & & 24 \\
\hline 9 & 3 & $\mathrm{Et}_{2} \mathrm{CO}$ & $\mathrm{PhCHO}$ & $3 \mathbf{i}$ & & 28 \\
\hline 10 & 4 & $\mathrm{Et}_{2} \mathrm{CO}$ & $\mathrm{PhCHO}$ & $3 \mathbf{j}$ & & 31 \\
\hline
\end{tabular}

${ }^{a}$ All products 3 were $>95 \%$ pure (GLC and/or $300 \mathrm{MHz}{ }^{1} \mathrm{H}$ NMR) and were fully characterized by spectroscopic means (IR, ${ }^{1} \mathrm{H}$ - and ${ }^{13} \mathrm{C}$ NMR, and LR- and HR mass spectrometry).

${ }^{\mathrm{b}}$ Isolated yields of compounds 3 after column chromatography (silica gel, hexane/ethyl acetate) based on the starting chlorothioether 5 .

${ }^{\mathrm{c}} \mathrm{A}$ ca. 4:1 mixture of diastereomers was obtained ( $75 \mathrm{MHz}{ }^{13} \mathrm{C}$ NMR). 


\section{Experimental Section}

General Procedures. All reactions were carried out under an atmosphere of argon in oven-dried glassware. All reagents were commercially available (Acros, Aldrich) and were used without further purification. Commercially available anhydrous THF $(99.9 \%$, water content $\leq 0.006 \%$, Acros) was used as solvent in all the lithiation reactions. IR spectra were measured (film) with a Nicolet Impact 400 D-FT Spectrometer. NMR spectra were recorded with a Bruker AC-300 or a Bruker ADVANCE DRX-500 using $\mathrm{CDCl}_{3}$ as the solvent. LRMS and HRMS were measured with Shimadzu GC/HS QP-5000 and Finningan MAT95 S spectrometers, respectively. The purity of volatile products and the chromatographic analyses (GLC) were determined with a flame ionisation detector and a $12 \mathrm{~m}$ capillary column $(0.2 \mathrm{~mm}$ diam., $0.33 \mu \mathrm{m}$ film thickness $)$, using nitrogen $(2 \mathrm{~mL} / \mathrm{min})$ as carrier gas, $T_{\text {injector }}=275^{\circ} \mathrm{C}, \mathrm{T}_{\text {detector }}=300{ }^{\circ} \mathrm{C}, \mathrm{T}_{\text {column }}=60^{\circ} \mathrm{C}(3$ $\min )$ and $60-270{ }^{\circ} \mathrm{C}\left(15{ }^{\circ} \mathrm{C} / \mathrm{min}\right), \mathrm{P}=40 \mathrm{kPa}$. Specific rotations were determined with a PerkinElmer 341 digital polarimeter.

\section{Preparation of 1-chloro-n-phenylsulfanylalkanes (5)}

Isolation of compounds 5. General procedure. Thiophenol (1.42 g, $21.0 \mathrm{mmol})$ was added to a solution of $\mathrm{KOH}(1.25 \mathrm{~g}, 22.5 \mathrm{mmol})$ in methanol $(40 \mathrm{~mL})$ at $0{ }^{\circ} \mathrm{C}$. After $10 \mathrm{~min}$, the corresponding 1-bromo-n-chloropropane $(20.0 \mathrm{mmol})$ was added, and stirring was continued at $20{ }^{\circ} \mathrm{C}$ for $4 \mathrm{~h}$. Then, the solvent was removed in a rotary evaporator, and the residue was hydrolyzed with water and extracted with ethyl acetate. The organic layer was dried over anhydrous magnesium sulfate and evaporated (15 Torr). The residue was purified by column chromatography (silica gel; hexane) to yield pure products $\mathbf{5}$. Yields, physical and spectroscopic data as well as literature references follow.

1-Chloro-2-phenylsulfanylethane $(\mathbf{5}, \mathbf{n}=\mathbf{0}) .{ }^{12}$ Pale yellow liquid, $68 \%$ yield; $R_{\mathrm{f}} 0.43$ (hexane); $v$ (film) $3071,3055 \mathrm{~cm}^{-1}(\mathrm{ArH}) ; \delta_{\mathrm{H}} 3.12\left(2 \mathrm{H}, \mathrm{t}, J=7.7 \mathrm{~Hz}, \mathrm{PhSCH}_{2}\right), 3.52(2 \mathrm{H}, \mathrm{t}, J=7.7 \mathrm{~Hz}$, $\left.\mathrm{ClCH}_{2}\right), 7.14-7.33(5 \mathrm{H}, \mathrm{m}, \mathrm{ArH}) ; \delta_{\mathrm{C}} 35.7,42.0\left(\mathrm{CH}_{2}\right), 126.6,128.9,129.9,134.1(\mathrm{ArC}) ; m / z 174$ $\left(\mathrm{M}^{+}, 21 \%\right), 172$ (62), 123 (100), 110 (18), 109 (27), 77 (12), 65 (18).

1-Chloro-3-phenylsulfanylpropane $(\mathbf{5}, \mathbf{n}=\mathbf{1}) .{ }^{13}$ Pale yellow liquid, $90 \%$ yield; $R_{\mathrm{f}} 0.37$ (hexane); $v$ (film) $3078,3056 \mathrm{~cm}^{-1}(\mathrm{ArH}) ; \delta_{\mathrm{H}} 2.06\left(2 \mathrm{H}, \mathrm{m}, J=6.6 \mathrm{~Hz}, \mathrm{CH}_{2} \mathrm{CH}_{2} \mathrm{CH}_{2}\right), 3.06(2 \mathrm{H}, \mathrm{t}$, $\left.J=7.0 \mathrm{~Hz}, \mathrm{PhSCH}_{2}\right), 3.65\left(2 \mathrm{H}, \mathrm{t}, J=6.3 \mathrm{~Hz}, \mathrm{ClCH}_{2}\right), 7.18-7.36(5 \mathrm{H}, \mathrm{m}, \mathrm{ArH}) ; \delta_{\mathrm{C}} 30.7,31.6$, $43.3\left(\mathrm{CH}_{2}\right), 126.2,128.9,129.5,135.6(\mathrm{ArC}) ; \mathrm{m} / \mathrm{z} 188\left(\mathrm{M}^{+}, 24 \%\right), 186(73), 123$ (100), 110 (86), 109 (24), 77 (15), 65 (21), 51 (23), 45 (60).

1-Chloro-4-phenylsulfanylbutane $(\mathbf{5}, \mathbf{n}=\mathbf{2}){ }^{12}$ Pale yellow liquid, $78 \%$ yield; $R_{\mathrm{f}} 0.25$ (hexane); $v$ (film) 3070, $3061 \mathrm{~cm}^{-1}(\mathrm{ArH}) ; \delta_{\mathrm{H}} 1.66-1.86\left[4 \mathrm{H}, \mathrm{m}, \mathrm{CH}_{2}\left(\mathrm{CH}_{2}\right)_{2} \mathrm{CH}_{2}\right], 2.85(2 \mathrm{H}, \mathrm{t}, J=6.8 \mathrm{~Hz}$, $\left.\mathrm{PhSCH}_{2}\right), 3.43\left(2 \mathrm{H}, \mathrm{t}, J=6.5 \mathrm{~Hz}, \mathrm{ClCH}_{2}\right), 7.10-7.31(5 \mathrm{H}, \mathrm{m}, \mathrm{ArH}) ; \delta_{\mathrm{C}} 25.8,31.0,32.2,44.0$ $\left(\mathrm{CH}_{2}\right), 125.3,128.4,128.5,136.1(\mathrm{ArC}) ; \mathrm{m} / z 202\left(\mathrm{M}^{+}, 22 \%\right), 200$ (64), 123 (100), $110(81), 109$ (19), 91 (32), 77 (14), 65 (17), 55 (26).

1-Chloro-5-phenylsulfanylpentane $(\mathbf{5}, \mathbf{n}=\mathbf{3}) .{ }^{12}$ Pale yellow liquid, $79 \%$ yield; $R_{\mathrm{f}} 0.20$ (hexane); $v$ (film) $3073,3055 \mathrm{~cm}^{-1}(\mathrm{ArH}) ; \delta_{\mathrm{H}} 1.50-1.72\left[6 \mathrm{H}, \mathrm{m}, \mathrm{CH}_{2}\left(\mathrm{CH}_{2}\right)_{3} \mathrm{CH}_{2}\right], 2.86(2 \mathrm{H}, \mathrm{t}, J=$ 
$\left.6.5 \mathrm{~Hz}, \mathrm{PhSCH}_{2}\right), 3.44\left(2 \mathrm{H}, \mathrm{t}, J=6.5 \mathrm{~Hz}, \mathrm{ClCH}_{2}\right), 7.12-7.28(5 \mathrm{H}, \mathrm{m}, \mathrm{ArH}) ; \delta_{\mathrm{C}} 25.7,28.1,31.8$, 33.0, $44.5\left(\mathrm{CH}_{2}\right), 125.4,128.5,128.6,136.5$ (ArC); m/z $216\left(\mathrm{M}^{+}, 21 \%\right), 214$ (60), 179 (28), 123 (72), 110 (100), 109 (22), 77 (13), 69 (41), 65 (17), 51 (14).

1-Chloro-2-phenylsulfanylhexane $(\mathbf{5}, \mathbf{n}=4) .{ }^{12}$ Pale yellow liquid, $91 \%$ yield; $R_{\mathrm{f}} 0.13$ (hexane); $v$ (film) $3074,3055 \mathrm{~cm}^{-1}(\mathrm{ArH}) ; \delta_{\mathrm{H}} 1.38-1.41\left(4 \mathrm{H}, \mathrm{m}, 2 \times \mathrm{CH}_{2}\right), 1.60-1.73\left(4 \mathrm{H}, \mathrm{m}, 2 \times \mathrm{CH}_{2}\right), 2.88$ $\left(2 \mathrm{H}, \mathrm{t}, J=7.2 \mathrm{~Hz}, \mathrm{PhSCH}_{2}\right), 3.46\left(2 \mathrm{H}, \mathrm{t}, J=6.6 \mathrm{~Hz}, \mathrm{ClCH}_{2}\right), 7.10-7.30(5 \mathrm{H}, \mathrm{m}, \mathrm{ArH}) ; \delta_{\mathrm{C}} 26.2$, 27.8, 28.7, 32.2, 33.2, $44.7\left(\mathrm{CH}_{2}\right), 125.5,128.6,136.7$ (ArC); $m / z 230\left(\mathrm{M}^{+}, 18 \%\right), 228(52), 193$ (14), 123 (33), 110 (100), 109 (17), 83 (11), 77 (10), 65 (12), 55 (15).

Selective monolithiation of 1-chloro-n-phenylsulfanylalkanes (6) and reaction with carbonyl compounds. Preparation of compounds 8.

Isolation of compounds 8. General procedure. To a cooled $\left(-78{ }^{\circ} \mathrm{C}\right)$ solution of the corresponding 1-chloro-n-phenylsulfanylalkane $(\mathbf{5}, 1.0 \mathrm{mmol})$ in THF $(2 \mathrm{~mL})$ was added dropwise a $0.7 \mathrm{M}$ THF solution of lithium-naphthalene $(3.2 \mathrm{~mL}, 2.2 \mathrm{mmol})$ and the reaction mixture was stirred at the same temperature for $20 \mathrm{~min}$. Then, the corresponding carbonyl compound $(1.1 \mathrm{mmol})$ was added dropwise at $-78{ }^{\circ} \mathrm{C}$ and after $10 \mathrm{~min}$ the reaction mixture was hydrolyzed with water $(4 \mathrm{~mL})$ and extracted with ethyl acetate $(3 \times 10 \mathrm{~mL})$. The organic layer was dried over anhydrous magnesium sulfate and evaporated (15 Torr). The residue was purified by column chromatography (silica gel; hexane/ethyl acetate) to yield pure product $\mathbf{8}$. Yields and structures are included in Table 1. Physical and spectroscopic data as well as literature references follow.

2,2-Dimethyl-6-phenylsulfanylhexan-3-ol (8a). Colourless oil; $R_{\mathrm{f}} 0.10$ (hexane/ethyl acetate: $10 / 1) ; v$ (film) 3570-3360 (OH), 3074, 3059, 2965, 2868, 1393, $1366 \mathrm{~cm}^{-1} ; \delta_{\mathrm{H}} 0.88[9 \mathrm{H}, \mathrm{s}$, $\left.\left(\mathrm{CH}_{3}\right)_{3} \mathrm{C}\right], 1.25-1.40\left(2 \mathrm{H}, \mathrm{m}, \mathrm{CH}_{2}\right), 1.60-1.72(1 \mathrm{H}, \mathrm{m}, \mathrm{CHH}), 1.75(1 \mathrm{H}$, br s, OH$), 1.86-1.99$ $(1 \mathrm{H}, \mathrm{m}, \mathrm{CH} H), 2.96\left(2 \mathrm{H}, \mathrm{t}, J=6.7 \mathrm{~Hz}, \mathrm{CH}_{2} \mathrm{SPh}\right), 3.16-3.19(1 \mathrm{H}, \mathrm{m}, \mathrm{CHOH}), 7.15-7.34(5 \mathrm{H}, \mathrm{m}$, $\operatorname{ArH}) ; \delta_{\mathrm{C}} 25.6\left(\mathrm{CH}_{3}\right), 26.6,30.4,33.7\left(\mathrm{CH}_{2}\right), 34.9(\mathrm{C}), 79.5(\mathrm{COH}), 125.7,128.8,129.0,136.7$ (ArC); $m / z 238\left(\mathrm{M}^{+}, 34 \%\right), 181$ (46), 163 (12), 136 (23), 135 (14), 123 (25), 110 (41), 109 (24), 77 (13), 71 (100), 57 (31), 45 (30); HRMS: $\mathrm{M}^{+}$, Found 238.1386. $\mathrm{C}_{14} \mathrm{H}_{22} \mathrm{OS}$ requires 238.1391.

1-(3-Phenylsulfanylpropyl)cyclohexanol (8b). Yellow oil; $R_{\mathrm{f}} 0.12$ (hexane/ethyl acetate: 10/1); $v$ (film) 3580-3210 (OH), 3054, 2930, 2854, 1447, $1265 \mathrm{~cm}^{-1} ; \delta_{\mathrm{H}} 1.42-1.67\left(15 \mathrm{H}, \mathrm{m}, 7 \times \mathrm{CH}_{2}\right.$, $\mathrm{OH}), 2.93\left(2 \mathrm{H}, \mathrm{t}, J=7.1 \mathrm{~Hz}, \mathrm{CH}_{2} \mathrm{SPh}\right), 7.15-7.35(5 \mathrm{H}, \mathrm{m}, \mathrm{ArH}) ; \delta_{\mathrm{C}} 22.2,22.7,25.7,29.7,34.3$, $37.4\left(\mathrm{CH}_{2}\right), 71.3(\mathrm{COH}), 125.75,128.8,129.0,136.7$ (ArC); m/z $250\left(\mathrm{M}^{+}, 12 \%\right), 136(100), 135$ (26), 123 (13), 110 (13), 81 (29), 67 (11), 55 (28), 45 (16), 43 (17), 41 (32); HRMS: M $^{+}$, Found 250.1389. $\mathrm{C}_{15} \mathrm{H}_{22} \mathrm{OS}$ requires 250.1391 .

7-Phenylsulfanyl-3-ethylheptan-3-ol (8c). ${ }^{14}$ Colourless oil; $R_{\mathrm{f}} 0.11$ (hexane/ethyl acetate: 10/1); $v$ (film) 3550-3345 (OH), 3076, 3060, 2964, 2936, $1470 \mathrm{~cm}^{-1} ; \delta_{\mathrm{H}} 0.84(6 \mathrm{H}, \mathrm{t}, J=7.5 \mathrm{~Hz}$, $\left.2 \times \mathrm{CH}_{3}\right), 1.17\left(1 \mathrm{H}\right.$, br s, OH) $, 1.37-1.48\left(8 \mathrm{H}, \mathrm{m}, 4 \times \mathrm{CH}_{2}\right), 1.60-1.67\left(2 \mathrm{H}, \mathrm{m}, \mathrm{CH}_{2}\right), 2.92(2 \mathrm{H}, \mathrm{t}, J$ $\left.=7.3 \mathrm{~Hz}, \mathrm{CH}_{2} \mathrm{SPh}\right), 7.12-7.33(5 \mathrm{H}, \mathrm{m}, \mathrm{ArH}) ; \delta_{\mathrm{C}} 7.7\left(\mathrm{CH}_{3}\right), 22.5,29.6,30.9,33.5,37.6\left(\mathrm{CH}_{2}\right)$, 74.4 (COH), 125.6, 128.7, 128.9, 136.7 (ArC); m/z $252\left(\mathrm{M}^{+}, 34 \%\right), 234$ (48), 124 (60), 123 (41), 113 (92), 110 (58), 109 (50), 95 (100), 87 (25), 69 (39), 57 (60), 55 (38). 
8-Phenylsulfanyl-3-ethyloctan-3-ol (8d). Colourless oil; $R_{\mathrm{f}} 0.13$ (hexane/ethyl acetate: 10/1); $v$ (film) 3580-3350 (OH), 3072, 3060, 2963, 2930, $1483 \mathrm{~cm}^{-1} ; \delta_{\mathrm{H}} 0.84\left(6 \mathrm{H}, \mathrm{t}, J=7.4 \mathrm{~Hz}, 2 \times \mathrm{CH}_{3}\right)$, 1.26-1.47 (11H, m, 5× $\left.\mathrm{CH}_{2}, \mathrm{OH}\right), 1.61-1.69\left(2 \mathrm{H}, \mathrm{m}, \mathrm{CH}_{2}\right), 2.91\left(2 \mathrm{H}, \mathrm{t}, J=6.8 \mathrm{~Hz}, \mathrm{CH}_{2} \mathrm{SPh}\right)$, 7.12-7.33 (5H, m, ArH); $\delta_{\mathrm{C}} 7.7\left(\mathrm{CH}_{3}\right), 22.9,29.1,29.4,30.9,33.5,38.0\left(\mathrm{CH}_{2}\right), 74.5(\mathrm{COH})$, 125.6, 128.7, 128.8, 136.9 (ArC); m/z $266\left(\mathrm{M}^{+}, 37 \%\right), 248$ (59), 165 (25), 136 (15), 127 (39), 123 (81), 110 (100), 109 (98), 97 (28), 83 (39), 69 (35), 67 (31), 57 (73), 55 (60); HRMS: M+ Found 266.1714. $\mathrm{C}_{16} \mathrm{H}_{26} \mathrm{OS}$ requires 266.1704 .

9-Phenylsulfanyl-3-ethylnonan-3-ol (8e). Colourless oil; $R_{\mathrm{f}} 0.15$ (hexane/ethyl acetate: 10/1); $v$ (film) 3565-3380 (OH), 3078, 3060, 2960, 2930, $1478 \mathrm{~cm}^{-1} ; \delta_{\mathrm{H}} 0.83\left(6 \mathrm{H}, \mathrm{t}, J=7.4 \mathrm{~Hz}, 2 \times \mathrm{CH}_{3}\right)$, $1.14\left(1 \mathrm{H}\right.$, br s, OH), 1.21-1.31 (4H, m, 2× $\left.\mathrm{CH}_{2}\right), 1.35-1.46\left(8 \mathrm{H}, \mathrm{m}, 4 \times \mathrm{CH}_{2}\right), 1.60-1.65(2 \mathrm{H}, \mathrm{m}$, $\left.\mathrm{CH}_{2}\right), 2.90\left(2 \mathrm{H}, \mathrm{t}, J=7.4 \mathrm{~Hz}, \mathrm{CH}_{2} \mathrm{SPh}\right), 7.14-7.32(5 \mathrm{H}, \mathrm{m}, \mathrm{ArH}) ; \delta_{\mathrm{C}} 7.7\left(\mathrm{CH}_{3}\right), 23.1,28.7,29.0$, 29.7, 30.9, 33.4, $38.0\left(\mathrm{CH}_{2}\right), 74.4(\mathrm{COH}), 125.5,128.7,128.8,136.9$ (ArC); $m / z 280\left(\mathrm{M}^{+}, 16 \%\right)$, 262 (31), 251 (19), 165 (18), 123 (100), 110 (67), 87 (20), 81 (26), 69 (24), 57 (36), 55 (46); HRMS: $\mathrm{M}^{+}$, Found 280.1853. $\mathrm{C}_{17} \mathrm{H}_{28} \mathrm{OS}$ requires 280.1861 .

\section{Sequential double lithiation of 1-chloro-n-phenylsulfanylalkanes (5) and reaction with electrophiles. Preparation of diols 3.}

Isolation of compounds 3. General procedure. To a cooled $\left(-78^{\circ} \mathrm{C}\right)$ solution of 1-chloro-nphenylsulfanylalkane $(5,1.0 \mathrm{mmol})$ in THF $(2 \mathrm{~mL})$ was added dropwise a $0.7 \mathrm{M}$ THF solution of lithium-naphthalene $(3.2 \mathrm{~mL}, 2.2 \mathrm{mmol})$ and the reaction mixture was stirred at the same temperature for $20 \mathrm{~min}$. Then, a first carbonyl compound $(1.1 \mathrm{mmol})$ was added dropwise at -78 ${ }^{\circ} \mathrm{C}$ and after $10 \mathrm{~min}$., lithium powder $(28 \mathrm{mg}, 4.0 \mathrm{mmol})$ was added at once. The resulting reaction mixture was stirred for $1.5 \mathrm{~h}$ at around $-50{ }^{\circ} \mathrm{C}$ and a second carbonyl compound (1.1 mmol) was added dropwise at the same temperature. After $15 \mathrm{~min}$, it was hydrolyzed with water $(4 \mathrm{~mL})$ and extracted with ethyl acetate $(3 \times 10 \mathrm{~mL})$. The organic layer was dried over anhydrous magnesium sulfate and evaporated (15 Torr). The residue was purified by column chromatography (silica gel; hexane/ethyl acetate) to yield pure products $\mathbf{3}$. Yields and structures are included in Table 2. Physical and spectroscopic data as well as literature references follow.

2,7,7-Trimethyloctane-2,6-diol (3a). ${ }^{15}$ White solid; mp $82-83{ }^{\circ} \mathrm{C}$ (dichloromethane/hexane); $R_{\mathrm{f}}$ 0.16 (hexane/ethyl acetate: 2/1); $v(\mathrm{KBr}) 3500-3100(\mathrm{OH}), 2935,1440 \mathrm{~cm}^{-1} ; \delta_{\mathrm{H}} 0.89[9 \mathrm{H}, \mathrm{s}$, $\left.\left(\mathrm{CH}_{3}\right)_{3} \mathrm{C}\right], 1.22\left[6 \mathrm{H}, \mathrm{s},\left(\mathrm{CH}_{3}\right)_{2} \mathrm{C}\right], 1.26-1.69\left(8 \mathrm{H}, \mathrm{m}, 3 \times \mathrm{CH}_{2}, 2 \times \mathrm{OH}\right), 3.21(1 \mathrm{H}, \mathrm{dd}, J=8.6,1.8$ $\mathrm{Hz}, \mathrm{CHOH}) ; \delta_{\mathrm{C}} 21.7,25.7,27.6\left(\mathrm{CH}_{3}\right), 29.1,29.3\left(\mathrm{CH}_{2}\right), 34.9(\mathrm{C}), 43.6\left(\mathrm{CH}_{2}\right), 71.0(\mathrm{COH}), 79.8$ $(\mathrm{CHOH}) ; m / z 170\left(\mathrm{M}^{+}-\mathrm{H}_{2} \mathrm{O}, 0.5 \%\right), 113$ (28), 95 (100), 82 (18), 69 (71), 56 (32), 43 (70).

2,2-Dimethyl-7-pentyldodecane-3,7-diol (3b). Colourless oil; $R_{\mathrm{f}} 0.51$ (hexane/ethyl acetate: $2 / 1)$; $v$ (film) 3490-3140 (OH), 2960, 2871, $1374 \mathrm{~cm}^{-1} ; \delta_{\mathrm{H}} 0.87\left(6 \mathrm{H}, \mathrm{t}, J=6.7 \mathrm{~Hz}, 2 \times \mathrm{CH}_{3}\right), 0.88$ [9H, s, $\left.\left(\mathrm{CH}_{3}\right)_{3} \mathrm{C}\right], 1.27-1.52\left(22 \mathrm{H}, \mathrm{m}, 11 \times \mathrm{CH}_{2}\right), 1.83(2 \mathrm{H}$, br s, $2 \times \mathrm{OH}), 3.19(1 \mathrm{H}, \mathrm{dd}, J=9.8,1.6$ $\mathrm{Hz}, \mathrm{CHOH}) ; \delta_{\mathrm{C}} 14.0,20.6,22.6\left(\mathrm{CH}_{3}\right), 23.1,23.2,25.7,31.8,32.4\left(\mathrm{CH}_{2}\right), 34.8(\mathrm{C}), 38.9,39.0$, $39.2\left(\mathrm{CH}_{2}\right), 74.4(\mathrm{COH}), 79.5(\mathrm{CHOH}) ; m / z 282\left(\mathrm{M}^{+}-\mathrm{H}_{2} \mathrm{O}, 2 \%\right), 211$ (25), 207 (19), 193 (19), 171 (29), 137 (24), 123 (29), 109 (46), 97 (38), 96 (38), 95 (55), 83 (36), 81 (50), 69 (64), 67 (46), 57 (88), 55 (100), 43 (98); HRMS: $\mathrm{M}^{+}-\mathrm{H}_{2} \mathrm{O}$, Found 282.2927. $\mathrm{C}_{19} \mathrm{H}_{38} \mathrm{O}$ requires 282.2923. 
5-Pentyl-1-phenyldecane-1,5-diol (3c). Pale yellow solid; $\mathrm{mp} \quad 71-73 \quad{ }^{\circ} \mathrm{C}$ (dichloromethane/hexane); $R_{\mathrm{f}} 0.39$ (hexane/ethyl acetate: $\left.2 / 1\right) ; v(\mathrm{KBr}) 3530-3130(\mathrm{OH}), 3063$, $3029,2970,2955,2861,1455 \mathrm{~cm}^{-1} ; \delta_{\mathrm{H}} 0.87\left(6 \mathrm{H}, \mathrm{t}, J=6.8 \mathrm{~Hz}, 2 \times \mathrm{CH}_{3}\right), 1.20-1.41(22 \mathrm{H}, \mathrm{m}$, $\left.10 \times \mathrm{CH}_{2}, 2 \times \mathrm{OH}\right), 1.62-1.80\left(2 \mathrm{H}, \mathrm{m}, \mathrm{CH}_{2} \mathrm{CHOH}\right), 4.63(1 \mathrm{H}, \mathrm{dd}, J=8.0,5.5 \mathrm{~Hz}, \mathrm{CHOH}), 7.18-$ $7.32(5 \mathrm{H}, \mathrm{m}, \mathrm{ArH}) ; \delta_{\mathrm{C}} 14.0\left(\mathrm{CH}_{3}\right), 19.6,22.5,23.0,23.1,32.4,38.8,38.9,39.1,39.4\left(\mathrm{CH}_{2}\right), 74.2$ $(\mathrm{CHOH}), 74.5(\mathrm{COH}), 125.8,126.9,128.3,144.8(\mathrm{ArC}) ; m / z 284\left(\mathrm{M}^{+}-2 \mathrm{H}_{2} \mathrm{O}, 14 \%\right), 231$ (55), 213 (33), 157 (22), 143 (21), 129 (22), 117 (100), 91 (63), 79 (16), 67 (25), 55 (49), 43 (51), 41 (56); HRMS: $\mathrm{M}^{+}-\mathrm{H}_{2} \mathrm{O}$, Found 302.2613. $\mathrm{C}_{21} \mathrm{H}_{34} \mathrm{O}$ requires 302.2610.

1-[(4-Hydroxy-5,5-dimethyl)hexyl]cyclohexanol $\quad$ (3d). ${ }^{15}$ White solid; $\mathrm{mp} \quad 58-60 \quad{ }^{\circ} \mathrm{C}$ (dichloromethane/hexane); $R_{\mathrm{f}} 0.18$ (hexane/ethyl acetate: 2/1); $v(\mathrm{KBr}) 3700-3120(\mathrm{OH}), 2940$, $1446 \mathrm{~cm}^{-1} ; \delta_{\mathrm{H}} 0.89\left[9 \mathrm{H}, \mathrm{s},\left(\mathrm{CH}_{3}\right)_{3} \mathrm{C}\right], 1.23-1.66\left(18 \mathrm{H}, \mathrm{m}, 8 \times \mathrm{CH}_{2}, 2 \times \mathrm{OH}\right), 3.21(1 \mathrm{H}, \mathrm{dd}, J=10.4$, $1.8 \mathrm{~Hz}, \mathrm{CHOH}) ; \delta_{\mathrm{C}} 20.3,22.3\left(\mathrm{CH}_{2}\right), 25.7\left(\mathrm{CH}_{3}\right), 25.8,31.6,34.9,37.3,37.6\left(\mathrm{CH}_{2}\right), 41.9(\mathrm{C})$, $71.5(\mathrm{COH}), 79.9(\mathrm{CHOH}) ; \mathrm{m} / z 210\left(\mathrm{M}^{+}-\mathrm{H}_{2} \mathrm{O}, 2 \%\right), 135$ (35), 99 (29), 96 (34), 81 (38), 79 (26), 69 (36), 67 (62), 57 (67), 54 (93), 43 (67), 41 (100).

1-[(4-Hydroxy-4-phenyl)butyl]cyclohexanol (3e). ${ }^{15}$ Pale yellow oil; $R_{\mathrm{f}} 0.19$ (hexane/ethyl acetate: $2 / 1) ; v\left(\right.$ film) $3650-3130(\mathrm{OH}), 3030,2925,1436 \mathrm{~cm}^{-1} ; \delta_{\mathrm{H}} 1.21-1.78\left(16 \mathrm{H}, \mathrm{m}, 8 \times \mathrm{CH}_{2}\right)$, $2.25(2 \mathrm{H}$, br s, $2 \times \mathrm{OH}), 4.64(1 \mathrm{H}, \mathrm{dd}, J=7.9,5.5 \mathrm{~Hz}, \mathrm{CHOH}) ; \delta_{\mathrm{C}} 19.1,22.15,25.7,37.2,37.4$, $39.5\left(\mathrm{CH}_{2}\right), 71.5(\mathrm{COH}), 74.3(\mathrm{CHOH}), 125.8,127.3,128.3,144.9(\mathrm{ArC}) ; m / z 230\left(\mathrm{M}^{+}-\mathrm{H}_{2} \mathrm{O}\right.$, 4\%), 130 (10), 121 (13), 116 (58), 114 (14), 108 (30), 107 (33), 105 (18), 98 (18), 96 (26), 81 (68), 79 (100), 77 (76), 67 (46), 65 (20), 55 (92), 53 (27), 43 (56), 41 (95).

1-[(4-Hydroxy-4-phenyl)butyl]cyclooctanol (3f). Pale yellow oil; $R_{\mathrm{f}} 0.16$ (hexane/ethyl acetate: $2 / 1)$; $v$ (film) 3660-3180 (OH), 3062, 3028, 2980, 2925, $1452 \mathrm{~cm}^{-1} ; \delta_{\mathrm{H}} 1.36-1.68(20 \mathrm{H}, \mathrm{m}$, $\left.10 \times \mathrm{CH}_{2}\right), 2.04(2 \mathrm{H}$, br s, $2 \times \mathrm{OH}), 4.65(1 \mathrm{H}, \mathrm{dd}, J=8.0,5.5 \mathrm{~Hz}, \mathrm{CHOH}) ; \delta_{\mathrm{C}} 19.4,22.25,22.3$, 24.9, 28.1, 28.2, 36.0, 36.3, 39.5, $40.9\left(\mathrm{CH}_{2}\right), 74.3(\mathrm{CHOH}), 74.9(\mathrm{COH}), 125.8,127.3,128.3$, 144.9 (ArC); m/z $258\left(\mathrm{M}^{+}-\mathrm{H}_{2} \mathrm{O}, 3 \%\right.$ ), 240 (71), 183 (44), 170 (42), 157 (22), 155 (18), 149 (33), 130 (41), 121 (28), 117 (100), 115 (44), 108 (39), 104 (80), 91 (97), 81 (56), 79 (71), 67 (77), 55 (58), 41 (94); HRMS: $\mathrm{M}^{+}-\mathrm{H}_{2} \mathrm{O}$, Found 258.1993. $\mathrm{C}_{18} \mathrm{H}_{26} \mathrm{O}$ requires 258.1984.

(1S,2S,5R)-1-[(4-Hydroxy-5,5-dimethyl)hexyl]-2-isopropyl-5-methylcyclohexanol

(3g).

Diastereomeric mixture: Pale yellow oil; $R_{\mathrm{f}} 0.69$ (hexane/ethyl acetate: $2 / 1$ ); $v$ (film) 3680-3260 $(\mathrm{OH}), 2952,2869,1456,1390,1773 \mathrm{~cm}^{-1} ; \delta_{\mathrm{H}} 0.86-1.14\left(12 \mathrm{H}, \mathrm{m}, 3 \times \mathrm{CH}_{3}, \mathrm{CH}_{2}, \mathrm{CH}\right), 0.89$ [9H, s, $\left.\left(\mathrm{CH}_{3}\right)_{3} \mathrm{C}\right], 1.25-1.77\left(13 \mathrm{H}, \mathrm{m}, 5 \times \mathrm{CH}_{2}, \mathrm{CH}, 2 \times \mathrm{OH}\right), 2.04-2.14(1 \mathrm{H}, \mathrm{m}, \mathrm{CH}), 3.18-3.25(1 \mathrm{H}, \mathrm{m}$, $\mathrm{CHOH}) ; \delta_{\mathrm{C}} 18.1\left(\mathrm{CH}_{3}\right), 20.45,20.5,21.4\left(\mathrm{CH}_{2}\right), 22.45,23.6\left(\mathrm{CH}_{3}\right), 25.5,25.7,28.05\left(\mathrm{CH}_{3}\right), 32.0$, 32.1, 34.9, 35.1, 41.1, 41.3, 46.7, $46.8\left(\mathrm{CH}_{2}\right), 47.8,48.0(\mathrm{CH}), 75.1,75.2(\mathrm{COH}), 79.8,79.9$ $(\mathrm{CHOH}) ; m / z 284\left(\mathrm{M}^{+}, 1 \%\right), 191$ (14), 181 (20), 155 (30), 149 (16), 135 (43), 121 (22), 109 (28), 96 (54), 95 (62), 93 (28), 81 (71), 69 (59), 57 (65), 55 (75), 43 (78), 41 (100); HRMS: ${ }^{+}-\mathrm{H}_{2} \mathrm{O}$, Found 284.2705. $\mathrm{C}_{18} \mathrm{H}_{36} \mathrm{O}_{2}$ requires 284.2715.

6-Ethyl-1-phenyloctane-1,6-diol (3h). Colourless oil; $R_{\mathrm{f}} 0.22$ (hexane/ethyl acetate: 2/1); $v$ (film) 3490-3260 (OH), 3087, 3054, 3022, 2968, 2941, 2870, $1456 \mathrm{~cm}^{-1} ; \delta_{\mathrm{H}} 0.82(6 \mathrm{H}, \mathrm{t}, J=7.4$ $\left.\mathrm{Hz}, 2 \times \mathrm{CH}_{3}\right), 1.25-1.46\left(10 \mathrm{H}, \mathrm{m}, 5 \times \mathrm{CH}_{2}\right), 1.68-1.83\left(2 \mathrm{H}, \mathrm{m}, \mathrm{CH} \mathrm{CHOH}_{2}, 2.16(2 \mathrm{H}\right.$, br s, $2 \times \mathrm{OH}), 4.64(1 \mathrm{H}, \mathrm{dd}, J=7.3,5.7 \mathrm{~Hz}, \mathrm{CHOH}), 7.24-7.34(5 \mathrm{H}, \mathrm{m}, \mathrm{ArH}) ; \delta_{\mathrm{C}} 7.7,7.75\left(\mathrm{CH}_{3}\right)$, 
23.2, 26.3, 30.8, 30.9, 38.0, $38.9\left(\mathrm{CH}_{2}\right), 74.5(\mathrm{CHOH}), 74.6(\mathrm{COH}), 125.8,127.4,128.4,144.6$ (ArC); m/z $232\left(\mathrm{M}^{+}-\mathrm{H}_{2} \mathrm{O}, 2 \%\right), 203$ (13), 185 (14), 146 (18), 131 (27), 130 (28), 129 (15), 117 (26), 105 (100), 97 (41), 91 (43), 87 (27), 79 (38), 77 (26), 57 (39); HRMS: $\mathrm{M}^{+}-\mathrm{H}_{2} \mathrm{O}$, Found 232.1855. $\mathrm{C}_{16} \mathrm{H}_{24} \mathrm{O}$ requires 232.1827 .

7-Ethyl-1-phenylnonane-1,7-diol (3i). Colourless oil; $R_{\mathrm{f}} 0.27$ (hexane/ethyl acetate: 2/1); $v$ (film) 3520-3370 (OH), 3085, 3050, 3027, 2970, 2935, 2860, $1460 \mathrm{~cm}^{-1} ; \delta_{\mathrm{H}} 0.83(6 \mathrm{H}, \mathrm{t}, J=7.5$ $\left.\mathrm{Hz}, 2 \times \mathrm{CH}_{3}\right), 1.22-1.45\left(12 \mathrm{H}, \mathrm{m}, 6 \times \mathrm{CH}_{2}\right), 1.65-1.81\left(2 \mathrm{H}, \mathrm{m}, \mathrm{CH} \mathrm{CHOH}_{2}, 2.07\right.$ (2H, br s, $2 \times \mathrm{OH}), 4.63(1 \mathrm{H}, \mathrm{t}, J=6.7 \mathrm{~Hz}, \mathrm{CHOH}), 7.10-7.33(5 \mathrm{H}, \mathrm{m}, \mathrm{ArH}) ; \delta_{\mathrm{C}} 7.7\left(\mathrm{CH}_{3}\right), 23.2,25.7,30.0$, 30.9, 38.0, $39.0\left(\mathrm{CH}_{2}\right), 74.5(\mathrm{CHOH}), 74.6(\mathrm{COH}), 125.8,127.4,128.3,144.8(\mathrm{ArC}) ; m / z 228$ $\left(\mathrm{M}^{+}-2 \mathrm{H}_{2} \mathrm{O}, 10 \%\right), 199$ (18), 157 (29), 143 (25), 130 (28), 129 (35), 124 (31), 117 (41), 111 (29), 107 (100), 104 (33), 95 (28), 91 (58), 79 (64), 77 (39), 69 (25), 57 (33), 55 (38); HRMS: M+$2 \mathrm{H}_{2} \mathrm{O}$, Found 228.1871. $\mathrm{C}_{17} \mathrm{H}_{24}$ requires 228.1878 .

8-Ethyl-1-phenyldecane-1,8-diol (3j). Colourless oil; $R_{\mathrm{f}} 0.34$ (hexane/ethyl acetate: 2/1); $v$ (film) 3530-3290 (OH), 3086, 3060, 3032, 2969, $2856 \mathrm{~cm}^{-1} ; \delta_{\mathrm{H}} 0.82\left(6 \mathrm{H}, \mathrm{t}, J=7.6 \mathrm{~Hz}, 2 \times \mathrm{CH}_{3}\right)$, 1.24-1.45 (14H, m, $\left.7 \times \mathrm{CH}_{2}\right), 1.65-1.80\left(2 \mathrm{H}, \mathrm{m}, \mathrm{CH}_{2} \mathrm{CHOH}\right), 2.06(2 \mathrm{H}$, br s, $2 \times \mathrm{OH}), 4.63(1 \mathrm{H}$, $\mathrm{dd}, J=6.6 \mathrm{~Hz}, \mathrm{CHOH}), 7.20-7.35(5 \mathrm{H}, \mathrm{m}, \mathrm{ArH}) ; \delta_{\mathrm{C}} 7.7\left(\mathrm{CH}_{3}\right), 23.2,25.7,29.4,30.1,30.9,38.0$, $39.0\left(\mathrm{CH}_{2}\right), 74.5(\mathrm{CHOH}), 74.6(\mathrm{COH}), 125.8,127.4,128.3,144.9(\mathrm{ArC}) ; m / z 231\left(\mathrm{M}^{+}-2 \mathrm{H}_{2} \mathrm{O}-\right.$ Et, 12\%), 219 (23), 203 (27), 202 (30), 191 (16), 178 (14), 165 (24), 105 (100), 77 (92), 51 (11); HRMS: $\mathrm{M}^{+}-2 \mathrm{H}_{2} \mathrm{O}$, Found 242.2031. $\mathrm{C}_{18} \mathrm{H}_{26}$ requires 242.2035.

\section{Acknowledgements}

This work was generously supported by the Spanish Ministerio de Educación y Ciencia (MEC; grant no. CTQ2004-01261) and the Generalitat Valenciana (GV; grants no. GRUPOS05/052 and GRUPOS05/058). A.A. thanks the University of Alicante for a predoctoral fellowship. We also thank MEDALCHEMY S.L. for a gift of chemicals, especially lithium powder.

\section{References and Footnotes}

1. For reviews, see: (a) Foubelo, F.; Yus, M. Trends Org. Chem. 1998, 7, 1. (b) Foubelo, F.; Yus, M. Curr. Org. Chem. 2005, 9, 459.

2. For monographs, see: (a) Wakefield, B. J. Organolithium Methods; Academic Press: London, 1988. (b) Sapse, A. M.; von Ragué Schleyer, P., Eds. Lithium Chemistry: A Theoretical and Experimental Overview; J. Wiley \& Sons: New York, 1995. (c) Gray, M.; Tinkel, M.; Snieckus, V. In Comprehensive Organometallic Chemistry II; Abel, E. W.; Stone, F. G. A.; Wilkinson, G.; McKillop, A., Eds.; Pergamon: Oxford, 1995; Vol. 11, pp. 1-92. (d) Clayden, J. Organolithiums: Selectivity for Synthesis; Pergamon: Oxford, 2002. (e) 
The Chemistry of Organolithium Compounds; Rappoport, Z.; Marek, I., Eds.; Wiley: Chichester, 2004.

3. (a) Screttas, C. G.; Micha-Screttas, M. J. Org. Chem. 1978, 43, 1064. (b) Screttas, C. G.; Micha-Screttas, M. J. Org. Chem. 1979, 44, 713. (c) For a review, see: Cohen, T.; Bhupathy, M. Acc. Chem. Res. 1982, 22, 152. (d) For a recent application of this stoichiometric lithiation reaction, see: Deng, K.; Bensari-Bouguerra, A.; Whetstone, J.; Cohen, T. J. Org. Chem. 2006, 71, 2360.

4. (a) Yus, M. Chem. Soc. Rev. 1996, 155. (b) Yus, M. In reference 2e, Vol 1, pp. 647-747. Last papers from our laboratory using this methodology: (c) Meléndez, J.; Alonso, F.; Yus, M. Tetrahedron Lett. 2006, 47, 1187. (d) Alonso, F.; Meléndez, J.; Soler, T.; Yus, M. Tetrahedron 2006, 62, 2264. (e) Alonso, F.; Meléndez, J.; Yus, M. Tetrahedron 2006, 62, 4814. (f) Abou, A.; Foubelo, F.; Yus, M. Arkivoc 2007, iv, 196.

5. Foubelo, F.; Abou, A.; Yus, M. Eur. J. Org. Chem. 2005, 5089.

6. Alonso, F.; Yus, M. Recent Res. Devel. Org. Chem. 1997, 1, 397.

7. Abou, A.; Foubelo, F.; Yus, M. Tetrahedron 2006, 62, 10417.

8. For a preliminary communication, see: Foubelo, F.; Yus, M. Tetrahedron Lett. 2000, 41, 5047.

9. See, for instance: Almena, J.; Foubelo, F.; Yus, M. Tetrahedron 1995, 51, 11883.

10. See, for instance, the especial issue of Tetrahedron Symposium in Print (Eds.: Nájera, C.; Yus, M.) devoted to "Functionalised Organolithium Compounds" Tetrahedron 2005, 61.

11. See, for instance: Yus, M.; Moreno, B.; Foubelo, F. Synthesis 2004, 1115.

12. Sonda, S.; Kawahara, T.; Katayama, K; Sato, N.; Asano, K. Bioorg. Med. Chem. 2005, 13, 3295 .

13. Zimmerman, H. E.; Thyagarajan, B. S. J. Am. Chem. Soc. 1960, 82, 2505.

14. Perlman, K. L.; DeLuca, H. F. Tetrahedron Lett. 1992, 33, 2937.

15. Foubelo, F.; Saleh, S. A.; Yus, M. J. Org. Chem. 2000, 65, 3478. 